\title{
Chronic obstructive pulmonary disease assessment test predicts postoperative complications in patients with lung cancer qualified for lobectomy
}

\author{
Stefan Wesołowski ${ }^{1}$, Tadeusz Orłowski², Paweł Bujnowski ${ }^{3}$ \\ 'Department of Respiratory Physiopathology, Institute of Tuberculosis and Lung Diseases, Warsaw, Poland \\ ${ }^{2}$ Department of Thoracic Surgery, Institute of Tuberculosis and Lung Diseases, Warsaw, Poland \\ ${ }^{3}$ Systems Research Institute, Polish Academy of Sciences, Warsaw, Poland
}

Introduction. Patients considered for radical surgery for lung cancer need a functional evaluation to identify those at increased risk of postoperative complications.

Material and methods. We performed an analysis of clinical data of 1214 patients who underwent a single lobectomy for lung cancer. To assess the risk of complications, we used the chronic obstructive pulmonary disease assessment test (CAT). Results. 254 pulmonary and 51 cardiovascular complications occurred in 216 (17.8\%) patients. In 204 patients with a CAT score $\geq 12$ complications occurred more often than in patients with a lower score $(26.5 \%$ vs. $16.0 \% ; p<0.001)$.

Conclusions. In patients undergoing lobectomy for lung cancer, pulmonary complications occurred much more frequently than cardiovascular complications. Patients with a CAT score $\geq 12$ had a higher rate of postoperative complications as compared to those with a lower score.

Key words: lung cancer, lung resection, preoperative evaluation, CAT

\section{Introduction}

The goal of preoperative decision making is to minimise postoperative complications. Knowing which clinical factors are associated with complications permits oncologists, pulmonologists or surgeons to assess which candidates are appropriate for major thoracic surgery. Patients with lung cancer qualified for curative lung resection need an assessment of their health status, as they frequently suffer from comorbidities, mainly chronic obstructive pulmonary disease (COPD) and cardiovascular disease [1, 2], which are considered significant prognostic factors $[3,4]$.
The joint European Respiratory Society/European Society of Thoracic Surgeons and the American College of Chest Physicians $[5,6]$ recommend a stepwise functional workup of lung resection candidates. The assessment begins with a cardiovascular evaluation using the thoracic revised cardiac risk index (ThRCRI), then pulmonary function tests are performed with the calculation of predicted postoperative lung function. Patients with reduced predictive postoperative lung function are referred for exercise tests.

Current guidelines endorse the thoracic revised cardiac risk index (ThRCRI), as a first-line screening tool assessing the

\section{How to cite:}

Wesołowski S, Orłowski T, Bujnowski P. Chronic obstructive pulmonary disease assessment test predicts postoperative complications in patients with lung cancer qualified for lobectomy. NOWOTWORY J Oncol 2021; 71: 220-224.

This article is available in open access under Creative Common Attribution-Non-Commercial-No Derivatives 4.0 International (CC BY-NC-ND 4.0) license, allowing to download articles and share them with others as long as they credit the authors and the publisher, but without permission to change them in any way or use them commercially. 
cardiac risk before lung resection [7]. The ThRCRI includes cardiac ischaemia, cerebrovascular disease, renal disease and the extent of resection (pneumonectomy). However, simple registration of comorbidities does not provide information on the impact of comorbidities on patients' health. Besides, an assessment of the risk of pulmonary complications is not included at this stage of the preoperative evaluation. The chronic obstructive pulmonary disease assessment test (CAT) was introduced in 2009 as a tool to measure health status impairment in patients with COPD [8]. The CAT is a simple, patient-completed questionnaire, that covers a broad range of effects of COPD on patients' health. It is a standardised assessment tool consisting of only 8 items, that provides reliable and valid information on symptoms, activity limitation and other manifestations of COPD. We assumed that the CAT could also measure the symptomatic effect of other comorbidities and be useful in assessing the risk of complications in all patients qualified for lung resection, regardless of whether they have COPD or not.

The current study aimed to:

- rate the incidence of pulmonary complications and evaluate the need to include these type of complications at an early stage of preoperative evaluation;

- determine the utility of incorporating the CAT in the initial stage of the overall risk assessment of perioperative pulmonary and cardiovascular complications in patients with lung cancer qualified for lobectomy.

\section{Materials and methods}

The study was accepted by the local ethics committee. The participating subjects were patients who had undergone a single lobectomy for lung cancer at the Department of Thoracic Surgery, Institute of Tuberculosis and Lung Diseases in Warsaw, between March 2014 and March 2019. Preoperative staging included a complete medical history and physical examination, blood and urine examinations, a 12-lead resting electrocardiogram, pulmonary functional tests (PFT), computed tomography of the chest, and a fibrobronchoscopy. In the presence of symptomatic or previously documented cardiovascular disease, echocardiography, neck or lower limb ultrasound studies were performed. PFT included spirometry with measurements of forced expiratory volume in one second $\left(F V_{1}\right)$ and forced vital capacity (FVC) and a lung transfer factor for carbon monoxide ( $T L_{c o}$ ) measurements using the single breath method.

Tests were performed using a MasterScreen system (software version 4.65; Jaeger, Würzburg, Germany). We used reference values from the 2012 Global Lung Function Initiative for spirometry [9] and from the 1993 ERS/European Community for Coal and Steel for $T L_{c o}[10]$. The spirometric criterion of COPD diagnosis was $F E V_{1} / F V C$ ratio $<0.7$ in line with the Global Initiative for Chronic Obstructive Lung Disease (GOLD) [11]. The classification of airflow limitation severity was also taken from the GOLD, mild $-\mathrm{FEV}_{1} \geq 80 \%$ predicted, moderate $-\mathrm{FEV} \mathrm{V}_{1}<80 \%$ and $\geq 50 \%$ predicted, severe $-F E V_{1}<50 \%$ and $\geq 30 \%$ predicted. The ThRCRI score was calculated according to Brunelli et al. [8].

In our study, the ThRCRI included only three classes, as patients undergoing pneumonectomy were not included in the analysis. The Polish version of the CAT, obtained from the website www.catestonline.org, was administered to patients while they were waiting for PFT. The patients were briefly informed on how to complete the questionnaire and then filled it in independently. The CAT consists of eight items assessing cough, phlegm, chest tightness, breathlessness going up a hill/ stairs, activity limitations at home, confidence in leaving home, sleep and energy, with 6-point ordinal scales (scored 0-5) of severity for each item, that provides a scoring range of 0-40.

Lobectomies were performed by either thoracotomy or video-assisted thoracoscopic surgery. Stage I tumours were removed using a minimally invasive technique. The surgical approach was decided by experienced thoracic surgeons. Extended lobectomies (e.g. chest wall resections) were excluded from the analysis. Complications and deaths were those occurring within 30 days postoperatively or later if the patient was still in the hospital. The following pulmonary and cardiovascular complications were included: prolonged air leak, respiratory failure requiring mechanical ventilation for more than 48 hours, atelectasis or retention of secretions in the airways requiring bronchoscopy, pneumonia, myocardial infarction, cardiac failure, pulmonary embolism and death of the patient. The degree of severity of complications was assigned according to the Clavien-Dindo classification [12]. Only life-threatening complications or those requiring surgical or endoscopic intervention (category $>2$ ) were included in the analysis.

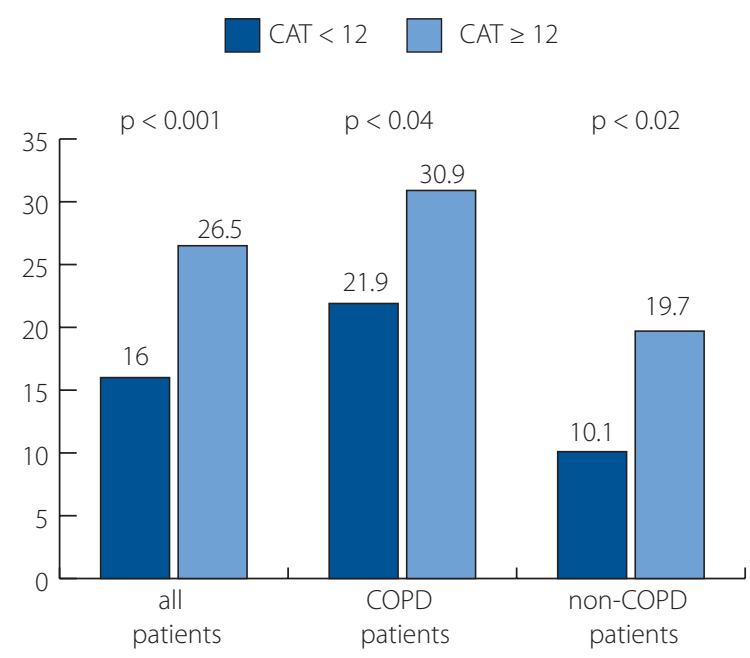

Figure 1. The incidence of complications by CAT score and COPD diagnosis 
Table I. Demographic and clinical characteristics of the study patients $(n=1214)$

\begin{tabular}{|lcccc|} 
& All patients & $\begin{array}{c}\text { COPD patients } \\
n=630\end{array}$ & $\begin{array}{c}\text { Non-COPD patients } \\
n=584\end{array}$ & p value \\
\hline age in years, mean (SD) & $65.2(8.6)$ & $66.4(7.6)$ & $63.9(9.3)$ & $<0.0001^{*}$ \\
\hline male, $n$ (\%) & $647(53)$ & $388(61.6)$ & $259(44.3)$ & $<0.0001^{* *}$ \\
\hline FEV $1^{\prime}$ \% predicted (SD) & $84.0(19.1)$ & $73.3(15.7)$ & $95.4(15.5)$ & $<0.0001^{*}$ \\
\hline FVC, \% predicted (SD) & $96.0(15.4)$ & $94.1(15.5)$ & $98.1(15.1)$ & $<0.0001^{*}$ \\
\hline FEV, /FVC, mean (SD) & $0.67(0.10)$ & $0.60(0.07)$ & $0.76(0.04)$ & $<0.001^{*}$ \\
\hline TL $_{\text {co } \% \text { predicted (SD) }}$ & $72.8(17.6)$ & $68.0(16.7)$ & $78.0(17.1)$ & $<0.0001^{*}$ \\
\hline CAT, mean (SD) & $6.4(5.7)$ & $6.8(6.0)$ & $5.9(5.5)$ & $0.006^{*}$ \\
\hline ThRCRI^, mean (SD) & $0.32(0.66)$ & $0.35(0.68)$ & $0.28(0.63)$ & $0.08^{*}$ \\
\hline comorbidity & & & & $0.28^{* *}$ \\
\hline COPD, $n$ (\%) & $630(51.9)$ & & $100(17)$ & $0.21^{* *}$ \\
\hline IHD, $n$ (\%) & $223(18)$ & $123(20 \%)$ & $10(2)$ & $0.72^{* *}$ \\
\hline CVD, $n$ (\%) & $29(2.4)$ & $19(3)$ & $2(0.4)$ & \\
\hline renal insufficiency, $n$ (\%) & $5(0.4)$ & $3(0.5)$ & & \\
\hline
\end{tabular}

Tests for COPD vs. non-COPD patients - * T-Test for means; ${ }^{*} \mathrm{Chi}^{2}$ test; $\mathrm{FEV}_{1}$ - forced expiratory volume in the first second; FVC - forced vital capacity; TL ${ }_{\text {CO }}-$ lung transfer factor for carbon monoxide; IHD - ischemic heart disease; CVD - cerebrovascular disease; SD - standard deviation; $\wedge$ - ThRCRI included only three items, without pneumonectomy (score range 0-4.5).

\section{Statistical analysis}

Categorical data were tested by the Chi² test. Tests for complications for CAT score $\geq 12$ vs. CAT score $<12$ and two more such tests included separately COPD and non-COPD patients (fig. 1) were adjusted using the Holm-Bonferroni method for multiple comparisons with $\mathrm{p}$ value thresholds adjusted to be respectively: 0.017, 0.025 and 0.05 . Lower test $p$-values were met in testing.

Numerical data statistics were presented in the form of means with standard deviations (SD). Examining table I, means of numerical variables for COPD and non-COPD groups were tested using the independent-samples T-Test. P values below 0.05 for 2-tailed tests were considered statistically significant to reject the hypothesis for equality. Analyses were performed using scientific computation libraries, SciPy (ver. 1.3) and Numpy (ver. 1.16.2), in the Python programming language (ver. 3.8).

\section{Results}

The demographic and clinical characteristics of the study subjects are shown in table I. Among 1214 subjects, 630 (52\%) patients met the spirometric criteria of the COPD diagnosis $($ FEV $1 / F V C<0.7)$, of whom 95\% were smokers, with mean pack-years 35.9 (SD: 21.9). There were 206 (33\%) cases with mild, 384 (61\%) moderate and 40 (6\%) with severe airway limitation. COPD was the most common comorbidity, followed by ischaemic heart disease. Cerebrovascular disease and renal insufficiency were rare, found in less than $3 \%$ of patients. The incidence of ischaemic heart disease, cerebrovascular disease and renal insufficiency was similar in COPD patients and non-COPD patients. The mean CAT score was 6.4 (SD: 5.7, range 0-32) and was higher in patients with COPD 6.8 (SD: 5.9) than in non-COPD patients 5.9 (SD: $5.5 ; p=0.005)$. Surgical
Table II. The distribution of patients in each class of the ThRCRI and the CAT

\begin{tabular}{|lcc|}
\hline & $\begin{array}{c}\text { Number of cases } \\
\mathbf{n}(\%)\end{array}$ & $\begin{array}{c}\text { Number of cases with } \\
\text { pulmonary and cardiovascular } \\
\text { complications } n(\%)\end{array}$ \\
\hline ThRCR ${ }^{*}$ & \\
\hline 0 & $972(80)$ & $164(16.9)$ \\
\hline $1-1.5$ & $224(18.5)$ & $50(22.2)$ \\
\hline $2-2.5$ & $5(0.5)$ & 0 \\
\hline$>2.5$ & $12(1)$ & $2(16)$ \\
\hline CAT & & $162(16.0)$ \\
\hline$<12$ & $1010(83)$ & $54(26.5)$ \\
\hline$\geq 12$ & $204(17)$ & \\
\hline
\end{tabular}

* - ThRCRI included only three items, without pneumonectomy (score range 0-4.5).

interventions comprised 434 (36\%) right upper, 288 (24\%) left upper, 210 (17\%) left lower, 193 (16\%) right lower, and 89 (7\%) middle lobe lobectomies.

From the entire group of 1214 patients, 235 pulmonary and 51 cardiovascular complications were registered, which occurred in 216 (17.8\%) patients. Grade 3a complications (surgical or endoscopic intervention not under general anaesthesia) occurred in 168 (13.8\%), grade 3b complications (surgical or endoscopic intervention under general anaesthesia) in 4 $(0.3 \%)$, grade $4 a$ (life-threatening single organ dysfunction) in $23(1.9 \%)$, grade $4 \mathrm{~b}$ (life-threatening multi organ dysfunction) in $12(1.0 \%)$, and grade 5 (the death of the patient) complications in $9(0.7 \%)$ patients.

Based on the analysis of the discrimination of CAT scores in relation to complications, patients were divided into two groups, CAT score $<12$ and $\geq 12$. At this CAT bound, the variable was more precise in terms of assessing complications, 
compared to other tested thresholds, and at the same time included a fairly large number of patients with complications. The distribution of patients in classes of the ThRCRI and the CAT, and the number of cardiovascular and pulmonary complications in each class are shown in table II.

In the analysed group, the maximum value of the ThRCRI could be 4.5, not 5.5, because pneumonectomy was an exclusion criterion. $20 \%$ of patients had ThRCRI greater than zero, mainly due to comorbid ischaemic heart disease. The rate of pulmonary and cardiovascular complications increased in patients with a ThRCRI score $\geq 1,21.5 \%$ vs. $16.9 \%$ (Chi ${ }^{2}$ test; $p=0.048)$. The incidence of complications by CAT score in the whole group, in COPD and non-COPD patients, is shown in figure 1.

There were 204 (16.8\% of the whole group) patients with a CAT score $\geq 12$, and in these patients, complications occurred more often than in patients with a lower score $(26.5 \% \mathrm{vs}$. $16.0 \%$, Chi $^{2}$ test; $\left.p<0.001\right)$. This difference was true both in COPD patients $(30,9 \%$ vs. $21,9 \%, p<0.04)$ and in non-COPD patients (19.7\% vs. 10.1\%, $\mathrm{p}<0.02)$. In COPD patients, the rate of complications increased with the severity of airway limitation, $16 \%, 27 \%$ and $30 \%$ in patients with mild, moderate and severe airway obstruction respectively.

\section{Conclusions}

Thoracic surgery is considered high-risk surgery but remains the best therapeutic option for a cure in patients with resectable non-small cell lung cancer. Patients with lung cancer who are considered for radical surgery should be assessed to identify subjects at increased risk of perioperative complications. The initial step in the current algorithm of functional qualification focused on cardiovascular complications has limitations. The incidence of major cardiac complications is low $-3.3 \%$ in the series by Brunelli et al. [8], 4.1\% in the series by Ferguson et al. [13] and in our group.

We found major pulmonary complications more than four times more common than cardiovascular complications, so the risk of this type of complication should be assessed from the beginning of the functional workup. Pulmonary function tests, which are the second step of the functional workup, allow for the calculation of predictive postoperative lung function, but they are not a good tool for health status assessment. Several studies have indicated that the relationship between lung function and health status scores is weak $[14,15]$. We used the CAT, a simple instrument to assess the symptomatic impact on patients' health. The CAT was developed as an instrument to measure the health status of patients with COPD in an easy way [9]. The CAT covers all the important symptomatic areas of COPD and consists of only 8 items that provides a quantified measure of health status.

The item selection process followed a vigorous methodology and created a questionnaire with very good measurement properties. It requires only 2-3 minutes to complete and score, which enables rapid and reliable assessment of a patient's health status that can be readily incorporated into routine care $[16,17]$. The CAT was extensively evaluated in COPD patients with varying severity of the disease across Europe [18]. In our opinion, symptoms assessed by CAT are not limited to COPD and many of them, e.g. breathlessness going up a hill/stairs, activity limitation at home, confidence in leaving home, sleep and energy levels, are common to lung and cardiovascular diseases. That was the reason we decided to use the CAT as a tool to assess the risk of pulmonary and cardiovascular complications in all patients qualified for lung resection. We found that patients with a CAT score $\geq 12$ had more complications than patients with a lower CAT score and the higher incidence of complications was independent of the COPD diagnosis. The CAT identified the high-risk group at a very early stage of functional workup before PFT or exercise tests were performed. It is difficult to compare the results of our study with other series because we have found only one study aimed at verifying the usefulness of symptoms or quality of life-based scores, including the CAT, in predicting the risk of pulmonary postoperative complications in patients with early-stage COPD [19].

Our study is the first in which the CAT was used in a large group of patients qualified for thoracic surgery regardless of COPD diagnosis. It is worth mentioning that the analysis in the current study differs from those by Brunelli et al. [7] and Ferguson et al. [13]. The series by Brunelli and Ferguson included patients who underwent lung resection for benign and malignant diagnoses, and our study group included only patients with lung cancer undergoing a single lobectomy - the most common type of resection (89\% of resection surgeries for lung cancer in our hospital). In this way, we got a large and homogeneous group of patients in terms of the extent of surgery, something we consider a strength of our study. Patients undergoing elective pneumonectomy were excluded from the analysis because we think these patients should undergo a separate functional workup, since such an extensive procedure is a very strong risk factor - independent of the accompanying diseases and the patient's state of health. In practice, in patients undergoing lobectomy, only ischaemic heart disease was a prevalent ThRCRI criterion, since renal insufficiency and cerebrovascular disease were rare, and found in less than 3\% of patients. Moreover, our analysis embraced not only cardiovascular, but also pulmonary perioperative complications, which were much more common. In this context, the CAT, which turned out to be more effective, could replace the THRCRI in the pre-operative functional assessment algorithm.

In conclusion, pulmonary complications, occurring much more frequently than cardiovascular complications, should be included in the risk assessment scheme for lung resection. The CAT turned out to be an effective and easy to apply in practice instrument for initial risk assessment in patients with lung can- 
cer qualified for lobectomy. Patients with a CAT score $\geq 12$ had a higher rate of pulmonary and cardiovascular complications as compared to those with a lower score. The CAT has enabled simple early identification of patients in the high-risk group who require the most thorough further functional assessment.

\section{Conflict of interest: none declared}

\section{Stefan Wesołowski}

Institute of Tuberculosis and Lung Diseases

Department of Respiratory Physiopathology

ul. Płocka 26

01-138 Warszawa, Poland

e-mail: s.wesolowski@igichp.edu.pl

Received: 15 Apr 2021

Accepted: 28 Apr 2021

\section{References}

1. López-Encuentra A. Comorbidity in operable lung cancer. Lung Cancer. 2002; 35(3): 263-269, doi: 10.1016/s0169-5002(01)00422-6.

2. Birim O, Kappetein AP, Bogers AdJ. Charlson comorbidity index as a predictor of long-term outcome after surgery for nonsmall cell lung cancer. Eur J Cardiothorac Surg. 2005; 28(5): 759-762, doi: 10.1016/j. ejcts.2005.06.046, indexed in Pubmed: 16157485.

3. Lüchtenborg $M$, Jakobsen $E$, Krasnik $M$, et al. The effect of comorbidity on stage-specific survival in resected non-small cell lung cancer patients. Eur J Cancer. 2012; 48(18): 3386-3395, doi: 10.1016/j. ejca.2012.06.012, indexed in Pubmed: 22795582

4. Ambrogi $V$, Pompeo E, Elia $\mathrm{S}$, et al. The impact of cardiovascular comorbidity on the outcome of surgery for stage I and II non-small-cell lung cancer1. Eur J Cardiothorac Surg. 2003; 23(5): 811-817, doi: 10.1016/ s1010-7940(03)00093-9.

5. Brunelli A, Charloux A, Bolliger CT, et al. European Respiratory Society and European Society of Thoracic Surgeons joint task force on fitness for radical therapy. ERS/ESTS clinical guidelines on fitness for radical therapy in lung cancer patients (surgery and chemo-radiotherapy). Eur Respir J. 2009; 34(1): 17-41, doi: 10.1183/09031936.00184308, indexed in Pubmed: 19567600.

6. Brunelli A, Kim AW, Berger KI, et al. Physiologic evaluation of the patient with lung cancer being considered for resectional surgery: Diagnosis and management of lung cancer, 3rd ed: American College of Chest Physicians evidence-based clinical practice guidelines. Chest. 2013; 143(5 Suppl): e166S-e190S, doi: 10.1378/chest.12-2395, indexed in Pubmed: 23649437.
7. Brunelli $A$ Varela $G$ Salati $M$ et al. Recalibration of the revised cardiac risk index in lung resection candidates. Ann Thorac Surg. 2010; 90(1): 199-203, doi: 10.1016/j.athoracsur.2010.03.042, indexed in Pubmed: 20609775.

8. Jones PW, Harding G, Berry P, et al. Development and first validation of the COPD Assessment Test. Eur Respir J. 2009; 34(3): 648-654, doi: 10.1183/09031936.00102509, indexed in Pubmed: 19720809.

9. Quanjer PH, Stanojevic S, Cole TJ, et al. ERS Global Lung Function Initiative. Multi-ethnic reference values for spirometry for the 3-95yr age range: the global lung function 2012 equations. Eur Respir J. 2012; 40(6): 1324-1343, doi: 10.1183/09031936.00080312, indexed in Pubmed: 22743675.

10. Cotes JE, Chinn DJ, Quanjer PH, et al. Standardization of the measurement of transfer factor (diffusing capacity). Report Working Party Standardization of Lung Function Tests, European Community for Steel and Coal. Official Statement of the European Respiratory Society. Eur Respir J Suppl. 1993; 16: 41-52, indexed in Pubmed: 8499053.

11. Global Initiative for Chronic Obstructive Lung Disease (GOLD)[internet] Global strategy for the diagnosis, management and prevention of chronic obstructive pulmonary disease. 2019. https://goldcopd.org/ gold-reports/ (10.04.2020).

12. Dindo D, Demartines N, Clavien PA. Classification of surgical complications: a new proposal with evaluation in a cohort of 6336 patients and results of a survey. Ann Surg. 2004; 240(2): 205-213, doi: 10.1097/01. sla.0000133083.54934.ae, indexed in Pubmed: 15273542.

13. Ferguson MK, Celauro AD, Vigneswaran WT. Validation of a modified scoring system for cardiovascular risk associated with major lung resection. Eur J Cardiothorac Surg. 2012; 41(3): 598-602, doi: 10.1093/ ejcts/ezr081, indexed in Pubmed: 22345181.

14. Jones PW. Health status measurement in chronic obstructive pulmonary disease. Thorax. 2001; 56(11): 880-887, doi: 10.1136/thorax.56.11.880, indexed in Pubmed: 11641515.

15. Agusti A, Calverley PMA, Celli B, et al. Evaluation of COPD Longitudinally to Identify Predictive Surrogate Endpoints (ECLIPSE) investigators. Characterisation of COPD heterogeneity in the ECLIPSE cohort. Respir Res. 2010; 11: 122, doi: 10.1186/1465-9921-11-122, indexed in Pubmed: 20831787.

16. Kelly JL, Bamsey $\mathrm{O}$, Smith $\mathrm{C}$, et al. Health status assessment in routine clinical practice: the chronic obstructive pulmonary disease assessment test score in outpatients. Respiration. 2012; 84(3): 193-199, doi: 10.1159/000336549, indexed in Pubmed: 22441322

17. Raghavan N, Lam YM, Webb KA, et al. Components of the COPD Assessment Test (CAT) associated with a diagnosis of COPD in a random population sample. COPD. 2012; 9(2): 175-183, doi: 10.3109/15412555.2011.650802, indexed in Pubmed: 22409441.

18. Jones PW, Brusselle G, Dal Negro RW, et al. Health-related quality of life in patients by COPD severity within primary care in Europe. Respir Med. 2011; 105(1): 57-66, doi: 10.1016/j.rmed.2010.09.004, indexed in Pubmed: 20932736.

19. Kim ES, Kim YT, Kang $\mathrm{CH}$, et al. Prevalence of and risk factors for postoperative pulmonary complications after lung cancer surgery in patients with early-stage COPD. Int J Chron Obstruct Pulmon Dis. 2016; 11:1317-1326, doi: 10.2147/COPD.S105206, indexed in Pubmed: 27366059. 